\title{
Referentes femeninos en la cultura arquitectónica española. Las revistas especializadas como herramienta de análisis para la valoración de la visibilidad del trabajo realizados por mujeres
} (1973-1990)

\author{
Female references in the Spanish architectural culture. The \\ specialized journals as an analytical tool for the assessment of the \\ visibility of work carried out by women (1973-1990)
}

\author{
Lucía C. Pérez-Moreno y Penélope Santamera ${ }^{2}$
}

Fecha de recepción: 29-03-2018 - Fecha de aceptación: 21-06-2018

Hábitat y Sociedad (ISSN 2173-125X), n. . 11, noviembre de 2018, pp. 31-47.

http://dx.doi.org/10.12795/HabitatySociedad.2018.i11.03

\begin{abstract}
This article proposes a statistical analysis of the presence of women in Spanish journals specialized in architecture during the years of the political 'transition' to democracy (1973-1982) and its consolidation (19821990). These years of political, social and cultural transformation are considered a period of change in Spanish architectural culture, being the integration of women into the profession and the dissemination of their work in the media one of its main characteristics. This analysis is complemented with reflections on the education of women during the years prior to the Spanish 'transition', and the evolution of their incorporation into the Schools of Architecture. The article proposes three main objectives: on the one hand, to study the dissemination of work carried out by women compared to that of their male colleagues; on the other hand, to analyse the way in which this generation of female professionals saw their work disclosed, whether it was done alone, as part of a team, or as collaborators in architectural offices; and, finally, to establish a first genealogy of outstanding female architects whose trajectory should be a subject for future academic studies, such us Carme Pinós, Roser Amadó, María Teresa Muñoz, and Martha Thorne.
\end{abstract}

\section{Key words}

Spanish architecture; Female education; Political transition; Women in architecture; Democracy

\section{Resumen}

Este artículo plantea un análisis estadístico sobre la presencia de mujeres en revistas españolas especializadas en arquitectura en los años de la 'transición' política a la democracia (1973-1982) y la consolidación de esta (1982-1990). Estos años de transformación política, social y cultural se consideran un periodo de cambio en la cultura arquitectónica española, siendo la incorporación de la mujer a la profesión y la diseminación de su trabajo en estos medios una de sus características destacadas. Este análisis se acompaña con una serie de reflexiones en torno a la educación de la mujer, en los años previos a la 'transición' española, y a su incorporación en los estudios universitarios de arquitectura. El artículo plantea tres objetivos principales: por un lado, estudiar la diseminación que tuvo el trabajo realizado por mujeres en comparación con el de colegas masculinos; por otro lado, analizar el modo en el que esta generación de profesionales vio su trabajo divulgado, si lo hizo en solitario, como parte de un equipo o como colaboradoras en estudios de arquitectura; y, finalmente, establecer una primera genealogía de referentes femeninos sobresalientes cuya trayectoria debería ser objeto de futuros estudios académicos, como por ejemplo Carme Pinós, Roser Amadó, María Teresa Muñoz o Martha Thorne.

\section{Pallabras clave}

Arquitectura española; Educación de la mujer; Transición política; Arquitectas; Democracia

1 Doctora en Arquitectura, Profesora Contratada Doctora (Área de conocimiento: Composición arquitectónica). Escuela de Ingeniería y Arquitectura, Universidad de Zaragoza. E-mail: lcperez@unizar.es.

2 Arquitecta. Escuela de Ingeniería y Arquitectura, Universidad de Zaragoza. E-mail: penelopesantamera@gmail.com. 


\section{Introducción}

Los estudios de género se originaron como un campo de investigación interdisciplinar centrado en diversos temas relacionados con el género como categoría. La primera ola feminista centró su debate en visibilizar a las mujeres como personas con derechos propios, siendo su capacidad para votar uno de los objetivos fundamentales del movimiento. Con la segunda ola feminista, se analizó en sistema de sexo-género dominante en diferentes culturas (Rubin, 1975) y, además, el debate se abrió al análisis paralelo de otros ejes de opresión hacia las mujeres, como la orientación sexual (Wittig, 1973), la edad, la raza o la clase social (Lorde, 1984), entre otros. Mientras la tercera ola feminista esencialmente viene cuestionando la propia categoría de mujer como sujeto del feminismo (Butlter, 1990). Desde hace unos años, algunas pensadoras sostienen que estamos viviendo una cuarta ola feminista (Munro, 2013) caracterizada por la diversidad de propósitos y por el papel que internet está teniendo en su proliferación internacional y aceptación social global.

Ciertamente, somos testigos de una larga serie de actividades cuyo objetivo principal es visibilizar el trabajo realizado por mujeres. En el caso de las disciplinas de la arquitectura y el urbanismo, muchas de estas actividades recuperan el legado de determinadas profesionales desoídas o silenciadas en las diferentes historias canónicas de la arquitectura y el urbanismo moderno, con el objetivo de contextualizar teóricamente su trabajo y estudiar su aportación histórica. Mientras, otras iniciativas analizan las realidades sociales en las que vivieron esas mujeres y las opresiones que sufrieron, lo cual supone estudiar sus respectivas culturas, momentos históricos y geografías. Estas actividades se están divulgando a través de formatos de investigación tradicionales, como son artículos académicos, libros, exposiciones o proyectos de investigación; pero en paralelo, y en plena consonancia con esa cuarta ola feminista, aumentan iniciativas a través de medios de mayor rapidez de difusión, como listados de arquitectas reseñables o breves entradas de biografías en redes sociales y plataformas digitales, como el blog "un día | una arquitecta", la web "Pioneering Women in American Architecture" o las plataformas Women in Architecture en España o Die Architektin en Alemania. En unas y otras hay un objetivo común: aumentar la presencia de referentes femeninos en la cultura arquitectónica, una tarea primordial para que la sociedad en general (y no solo la occidental) y las jóvenes generaciones de profesionales puedan entender y valorar estas disciplinas en términos de igualdad. Así, introducir la perspectiva de género en el ámbito de la arquitectura y el urbanismo abre un campo de trabajo de alto valor antropológico que aporta nuevos enfoques de análisis tanto para la investigación académica como para la transferencia de sus resultados a la sociedad.

Este artículo se plantea como un paso más en la presente producción de investigaciones y actividades que analizan cómo ha sido la evolución y la incorporación de la mujer en la disciplina de la arquitectura y el urbanismo. Concretamente, se pretende analizar un momento histórico y geográfico determinado, los años de la ‘transición' política y la consolidación de la democracia en España.

El proceso de transformación política, social y cultural que sufrió España en el periodo denominado como 'transición' aparece como motivo temático y marco temporal de trabajo en ámbitos como la historia y el arte, entre otros. Numerosas monografías y artículos cientí- 
ficos analizan los cambios que vivió la cultura española atendiendo a ámbitos como el cine (Arnell y García Castañeda, 1988), la televisión (Palacio, 2011) o la literatura (Ansón, 2010), entre otros; mientras, la arquitectura es un ámbito cultural escasamente explorado. La incorporación de la mujer a esta disciplina es uno de los cambios más significativos que tuvieron lugar en estos años en esta profesión, una cuestión que no se contempla como tal en las historias de la arquitectura española del siglo xx. Así, el objetivo de esta investigación es analizar qué mujeres vieron su trabajo reconocido en estos años y de qué modo ejercieron la profesión - si lo hicieron en solitario, como parte de equipos o como colaboradoras- para con ello establecer una primera genealogía de referentes femeninos en la cultura arquitectónica española cuyo trabajo sea objeto de futuras investigaciones.

\section{La 'transición' política hacia la democracia como punto de inflexión para la cultura arquitectónica española}

Desde la historiografía de la arquitectura española del siglo xx, los años setenta son entendidos como años de cambio. Antón Capitel (1995), en "Arquitectura Española 1939-1992", da algunas claves para entender porqué (cfr. p. 481). Por un lado, habla de un cambio en el modo de pensar de los arquitectos, una "refundación disciplinar" cimentada desde la Escuelas de Arquitectura, verdaderos lugares de reflexión, como respuesta a la crisis de la modernidad, siendo los protagonistas una nueva generación de arquitectos que terminaron la carrera en la última década del Régimen; una generación con una educación diferente a la anterior y con unas perspectivas de futuro también muy diferentes. Según Capitel, "el cambio iniciado en la cultura arquitectónica española durante los primeros años de la década de los setenta (...) significó más el rechazo del pensamiento moderno convencional (...) que el de muchas de sus diversas arquitecturas o de su propia tradición" (ob. cit., p. 481). A este cambio de tendencia se sumó el aumento de la escuelas de arquitectura y su masificación, potenciada por el Plan de Estudios del 64, que rebajaba los cursos académicos de 7 a 5 años, y, con ello, la definitiva ruptura del dominio de los focos de Madrid y Barcelona, dando entrada a otras escuelas como las de Sevilla, Navarra, Galicia y País Vasco como nuevos focos culturales. Según Capitel, se alcanzó un periodo de madurez en la cultura arquitectónica española que se caracterizó por la "convivencia de tendencias dispares, que aspiraron a la calidad en sus diferentes ambiciones sin pretender restar legitimidad a las demás", gracias en gran parte a la creciente "culturización universitaria" (ob. cit., pp. 483-484), consecuencia, asimismo, del auge editorial de la época y del éxito de este por el aumento de alumnado interesado en la historia, la teoría y la crónica de la arquitectura.

Asimismo, es importante tener en cuenta que en los años setenta, a la masificación de las universidades se sumó la progresiva y creciente incorporación de la mujer a los estudios de arquitectura, de tal modo que en estos años de la 'transición' nos encontramos con una primera generación de profesionales femeninas cuya obra arquitectónica se verá diseminada en medios especializados, y que eclosionó a finales de los años setenta y los años ochenta, como es el caso de Roser Amadó. ${ }^{3}$ Igualmente, y en plena consonancia con el auge editorial de la época, se comenzaron a publicar artículos sobre arquitectura con autoría fe-
3 Cabe resaltar que Roser Amadó fue una de las escasas arquitectas cuyo nombre aparece de manera explícita en los textos históricos de Antón Capitel. 
menina en revistas especializadas españolas. Esta cuestión no aparece señalada como un cambio relevante en la cultura arquitectónica española en textos escritos en los años noventa, como es el caso del de Capitel; una cuestión que, sin embargo, merece estudios detallados.

\section{Realidad social de la educación de la mujer y su incorporación a los estudios de arquitectura}

Como apuntan los estudios de Inés Sánchez de Madariaga (2012), Matilde Ucelay fue la primera mujer en obtener el título de arquitecta en Madrid en 1936, coincidiendo con el final de la Segunda República (1931-1939) (Vilchez, 2012) y, por tanto, con el comienzo de la Guerra Civil (1936-1939). En estos años, había únicamente dos escuelas de arquitectura en España, una en Madrid y otra en Barcelona. Antes de la Guerra Civil, tan solo otras tres mujeres estudiaron arquitectura en Madrid; estas fueron: Lali Úrcola, que no llegó a terminar la carrera, Cristina Gonzalo y Rita Fernández-Queimadelos, tituladas en 1940 y 1941 respectivamente. En las dos décadas siguientes sólo otras cinco mujeres estudiaron arquitectura: en 1945 se tituló Margarita Mendizábal, en 1957 María Eugenia Pérez Clemente y, en 1958, Elena Arregui, las tres en Madrid (Agudo Arroyo y Sánchez de Madariaga, 2011). En los años sesenta, la escuela de Barcelona expidió su primer título a una mujer, Mercedes Serra Barenys, en 1964. Dos años antes, Margarita Brender Rubira convalidó sus estudios ya realizados por los de arquitectura en Barcelona, por lo que no se suele considerar que fuese la primera arquitecta catalana, ya que su formación no se desarrolló completamente en esa escuela (Muxí, 2013). Al final de esta década, y coincidiendo con el desarrollo económico de España, el número de arquitectas se incrementó hasta unas cuarenta.

Desafortunadamente, durante casi cuatro décadas, la educación de las mujeres en suelo español no fue la misma que la de los hombres, pues el régimen de Francisco Franco (1939-1975) propició una educación desigual para unos y otros. Desde el ámbito de la historia social, se señalan varias razones por las que el acceso de mujeres a los estudios universitarios fuese tan bajo en los años del régimen y la 'transición'. Según la historiadora María Ángeles Larumbe, la ideología impulsada por el régimen franquista, con el apoyo de la iglesia católica y de la Sección Femenina de Falange, promulgaba una educación discriminatoria para la mujer que alejó a estas de la universidad y del mundo laboral. El Régimen derogó "algunas leyes que la Segunda República había promulgado y que habían supuesto un avance cualitativo en la consideración igualitaria de las mujeres como sujeto de derecho" (Larumbe, 2004, pp. 17-18), lo que supuso que la sociedad de la época empujara a las mujeres a limitar sus expectativas profesionales anteponiendo el matrimonio y el cuidado de hijos e hijas, mayores y marido a la educación universitaria y al desarrollo profesional. Como señala Larumbe: "se consideraba que una buena situación familiar debía permitir que la esposa, y la hija, no trabajase, como muestra de la capacidad del varón de garantizar el sustento familiar" (ob. cit., pp. 28-29).

En 1974, la revista Triunfo publicaba un estudio sobre "El trabajo de la mujer en España" (Abril Navarro y Maranda López, 1974) donde se apuntaba que el porcentaje de mujeres en escuelas técnicas en el curso $1970-71$ era de 1,2\% frente al 43,3\% de hombres. Este desequilibrio académico se extendió consecuentemente al mundo laboral, y profe- 
siones técnicas como la arquitectura y la ingeniería estuvieron dominadas por profesionales masculinos. Asimismo, aunque el número de universitarias crecía de manera constante - en el curso 1960-61 hubo 13.778 mujeres universitarias y en el 1970-71 la cifra subió a 55.066, según los datos del Instituto Nacional de Estadística-, la mayoría de las mujeres que cursaban estudios universitarios eligieron profesiones socialmente relacionadas con su condición femenina, como magisterio o enfermería. Por ejemplo, en el curso 1970-71, el 56,5\% de estudiantes de magisterio fueron mujeres y el $84,9 \%$ lo fueron de enfermería. No obstante, los años setenta fueron un punto de inflexión considerable. Como señala Sánchez de Madariaga (2010), en el curso académico de 1978-79 el número de mujeres en las aulas de Madrid había ascendido hasta el 15\% — 357 mujeres frente a 2221 hombres—; un número que a lo largo de las siguientes tres décadas ascendería progresivamente llegando a la paridad en 2007, en el conjunto del país.

Además de esta inflexión en la incorporación de la mujer a los estudios de arquitectura, los años setenta se caracterizan por la proliferación de voces críticas ante la situación de desigualdad de la mujer en la sociedad española del momento. Las reivindicaciones de grupos españoles feministas tuvieron su mayor eclosión durante los años de la 'transición' política, es decir, desde la muerte de Francisco Franco en 1975 hasta la consolidación del primer gobierno democrático en 1982. Larumbe (2004) señala dos focos principales de reivindicación de los derechos de la mujer en estos años: la investigación académica y el ensayo de divulgación (cfr. ob. cit., pp. 45-62). Una de las pioneras en analizar aspectos jurídicos, sociológicos y antropológicos sobre la situación de la mujer en el Régimen fue Lidia Falcón, líder del Partido Feminista de España (PFE), directora de la revista Vindicación Feminista (1976-1979) y autora de numerosos estudios como: Los derechos civiles de la mujer (1963) o Los derechos laborales de la mujer (1964), entre otros. Asimismo, revistas culturales de la época se comprometieron con la causa y algunas, como Cuadernos para el Diálogo o Triunfo, publicaron números dedicados a difundir las circunstancias culturales y sociales en las que se desenvolvía la vida de las mujeres.

En paralelo a escritos reivindicativos, se fueron publicando traducciones al castellano de textos fundamentales de la literatura feminista como La mística de la feminidad de Betty Friedan o El segundo sexo de Simone de Beauvouir, ambos traducidos al castellano y publicados en 1966, lo que ayudó a crear un ambiente generalizado de protesta. A las reivindicaciones escritas se sumó la creación de asociaciones de mujeres, algunas de ellas clandestinas, que lucharon de manera activa porque sus derechos se viesen reconocidos, como la ASMSL (Asociación Nacional de Mujeres Separadas Legalmente), creada en 1973, o la ACMS (Asociación Católica de Mujeres Separadas) y el PFE (Partido Feminista de España), fundado en 1975, entre otros (cfr. Larumbe, 2002, p. 167). A partir de este año, tras la muerte de Francisco Franco, se sucedieron un gran número de reuniones, asambleas y actos que lucharon por recuperar derechos perdidos durante el Régimen y lograr otros nuevos, como la despenalización del aborto y del adulterio, la legalización de los anticonceptivos o, simplemente, el poder abrir una cuenta bancaria de manera autónoma. Todo esto fue crucial para que las mujeres pudiesen decidir sobre sus propias vidas y desarrollar una carrera profesional propia, autónoma e independiente económicamente.

En paralelo a este ambiente de reivindicación social, especialmente vibrante en Madrid y Barcelona, el número de mujeres que estudiaban 
arquitectura iba constantemente creciendo. La primera generación de arquitectas educadas en los últimos años del tardofranquismo y/o en la 'transición' fueron las primeras que pudieron ejercer la profesión en democracia (Pérez-Moreno, 2016). A pesar de que en su educación no contaron con referentes femeninos - pues en las escuelas de arquitectura de estos años los profesores seguían siendo hombres y los arquitectos modélicos también lo eran-, ellas fueron las primeras que pudieron abrirse camino en una sociedad con unos valores en evolución hacia la equidad. Todas ellas, todavía, pertenecían a una generación donde ser mujer y dedicarse a la arquitectura era algo inusual.

\section{Auge editorial y nuevas revistas de arquitectura especializadas}

En lo que se refiere al ámbito cultural especializado en arquitectura, a partir de 1975 comenzaron a publicarse nuevas revistas de arquitectura que ampliaron considerablemente el mercado editorial existente en los años anteriores. Los dos focos culturales predominantes - Madrid y Barcelona, derivados del ambiente universitario y cultural de sus respectivas Escuelas de Arquitectura- dominaron el panorama cultural español y la mayoría de las revistas de nueva creación tuvieron su base en estas ciudades. No obstante, se siguieron publicando revistas ligadas a instituciones relevantes del país, como la revista Arquitectura (iniciada en 1941 con el nombre de Revista Nacional de Arquitectura como revista oficial del Colegio de Arquitectos de Madrid), Cuadernos de Arquitectura (iniciada en 1944 como revista oficial del Colegio de Arquitectos de Cataluña; en 1971 pasaría a denominarse Cuadernos de Arquitectura y Urbanismo, y a partir de 1981 Quaderns d'arquitectura i urbanisme) o Informes de la Construcción (iniciada en 1948 y ligada al Instituto de la Construcción y el Cemento). Mientras, otras revistas de gran relevancia durante el Régimen, como Hogar y Arquitectura (revista oficial de la Obra Sindical del Hogar), desaparecieron con el cambio político.

La edición de nuevas publicaciones conllevó, además, enfoques editoriales diferentes: revistas como Arquitecturas Bis (Barcelona, 19741985), fueron más teóricas y dejaron en un segundo plano los tradicionales reportajes fotográficos de obras de arquitectura construidas; otras, como Carrer de la Ciutat (Barcelona, 1977-1980) o Ciudad y Territorio (Madrid, 1975), se centraron en temáticas relativas a la ciudad y el planeamiento urbano. En los años ochenta, y desde el ámbito de Madrid, aparecieron otras revistas que, en este caso, mantuvieron una línea editorial ligada al análisis de la crónica de arquitectura y a la edificación construida, entre las que destacan por su actual relevancia internacional AV Monografias (1987), Arquitectura Viva (1988) o El Croquis (1988). Todas ellas sirvieron de plataforma para reflexionar sobre la cultura arquitectónica del momento y para divulgar el trabajo realizado por los profesionales españoles y extranjeros.

\section{Planteamiento del análisis estadístico y resultados más significativos}

Con todo, el análisis de los artículos publicados en revistas españolas en estos años se presenta como una herramienta para datar la visibilidad y la relevancia que tuvo el trabajo realizado por mujeres. Como pri- 
mer acercamiento a esta temática, se han seleccionado seis revistas de arquitectura de especial relevancia para realizar un estudio estadístico: dos de mayor tradición, la revista Arquitectura y la revista Cuadernos de Arquitectura y Urbanismo, ambas ligadas a los Colegios de Arquitectos de sus respectivas ciudades, Madrid y Barcelona; y cuatro de reconocido impacto cultural, Arquitecturas Bis, AV Monografias, Arquitectura Viva y El Croquis. La cronología de análisis se ha limitado a los artículos publicados en cada una de ellas entre 1973 y 1990, es decir, los años de la 'transición' (1973-1982) y la consolidación de esta (1982-1990). ${ }^{4}$

Como muestra el Cuadro 1, el periodo estudiado se inicia con la presencia prácticamente inexistente de profesionales mujeres. El conjunto de revistas analizadas suman un total de 6.723 artículos publicados, de los cuales 489 cuentan con autoría femenina, es decir un 7,27\%. De las diferentes revistas, El Croquis destaca por ser la revista con mayor presencia de mujeres en sus páginas, con un total de 124 artículos, lo que supone un $14,5 \%$. En el lado opuesto se sitúa la revista $A r$ quitectura Bis, con un $3,82 \%$ de presencia femenina.

\begin{tabular}{|l|c|c|c|}
\hline Revista & n. ${ }^{\circ}$ total de artículos & $\begin{array}{l}\text { n. }{ }^{\circ} \text { de artículos } \\
\text { con presencia femenina }\end{array}$ & porcentaje \\
\hline Arquitectura & 1633 & 229 & 14,02 \\
\hline Cuadernos de Arquitectura y Urbanismo & 2574 & 231 & 8,97 \\
\hline Arquitecturas Bis & 785 & 30 & 3,82 \\
\hline El Croquis & 850 & 125 & 14,70 \\
\hline AV Monografías & 495 & 58 & 11,71 \\
\hline Arquitectura Viva & 386 & 45 & 11,65 \\
\hline
\end{tabular}

Como muestran las Figuras de $\mathbf{1}$ a 6 , a lo largo de los primeros años de la 'transición', la presencia de mujeres se sitúa entre el $0 \%$ y el $9 \%$, mientras que es a partir de 1982 cuando este porcentaje comienza a ascender, aunque no de manera homogénea. Estos datos tienen una cierta coherencia con el contexto político y la realidad social del país. Aunque es a principios de los años sesenta cuando la mujer comienza a incorporarse de manera progresiva a los estudios universitarios, no será hasta finales de los años setenta cuando se produzca cierto salto cuantitativo en su presencia en estos medios. Así, los despuntes en la visibilidad que aparecen en las diferentes figuras a principios de los ochenta podrían corresponder con el hecho de que el trabajo de estas arquitectas y urbanistas estuviese comenzando a adquirir cierta relevancia, coincidiendo con sus primeros años de actividad y experiencia laboral. En la revista Arquitectura, es el año 1987 el que sobresale (Figura 1), mientras en la revista del Colegio de Arquitectos de Cataluña destacan los años 1985 y 1990 (Figura 2). En el caso de la revista El Croquis (Figura 4) se alcanzan valores relevantes los años 1984 y 1986, mientras que en $A V$ Monografias (Figura 5) y en Arquitectura Viva (Figura 6) la presencia de mujeres va aumentando progresivamente y alcanza su valor más alto en 1990.
Cuadro 1: Número de artículos y porcentaje de ellos con presencia femenina en las diferentes revistas analizadas. Fuente: Elaboración propia.
4 Una versión preliminar sobre la visibilidad de arquitectas españolas en estas revistas fue presentada en una ponencia en el MoMoWo $3^{\text {rd International Conference- }}$ Workshop, Women Designers, Architects and Civil Engineers between 1969-1989, celebrado en Oviedo entre el 2 y el 4 de octubre de 2017. Este artículo profundiza en este análisis y amplia el estudio estadístico al trabajo realizado por profesionales extranjeras en revistas españolas. 
Figura 1: Presencia de arquitectos y arquitectas en la revista Arquitectura, del Colegio de Arquitectos de Madrid, entre 1973 y 1990 . Fuente: Elaboración propia.

Figura 2: Presencia de arquitectos y arquitectas en la revista Cuadernos de Arquitectura y Urbanismo/ Quaderns d'arquitectura i urbanisme, del Colegio de Arquitectos de Cataluña, entre 1973 y 1990. Fuente: Elaboración propia.

Figura 3: Presencia de arquitectos y arquitectas en la revista Arquitecturas Bis entre 1974 y 1985 . Fuente: Elaboración propia.
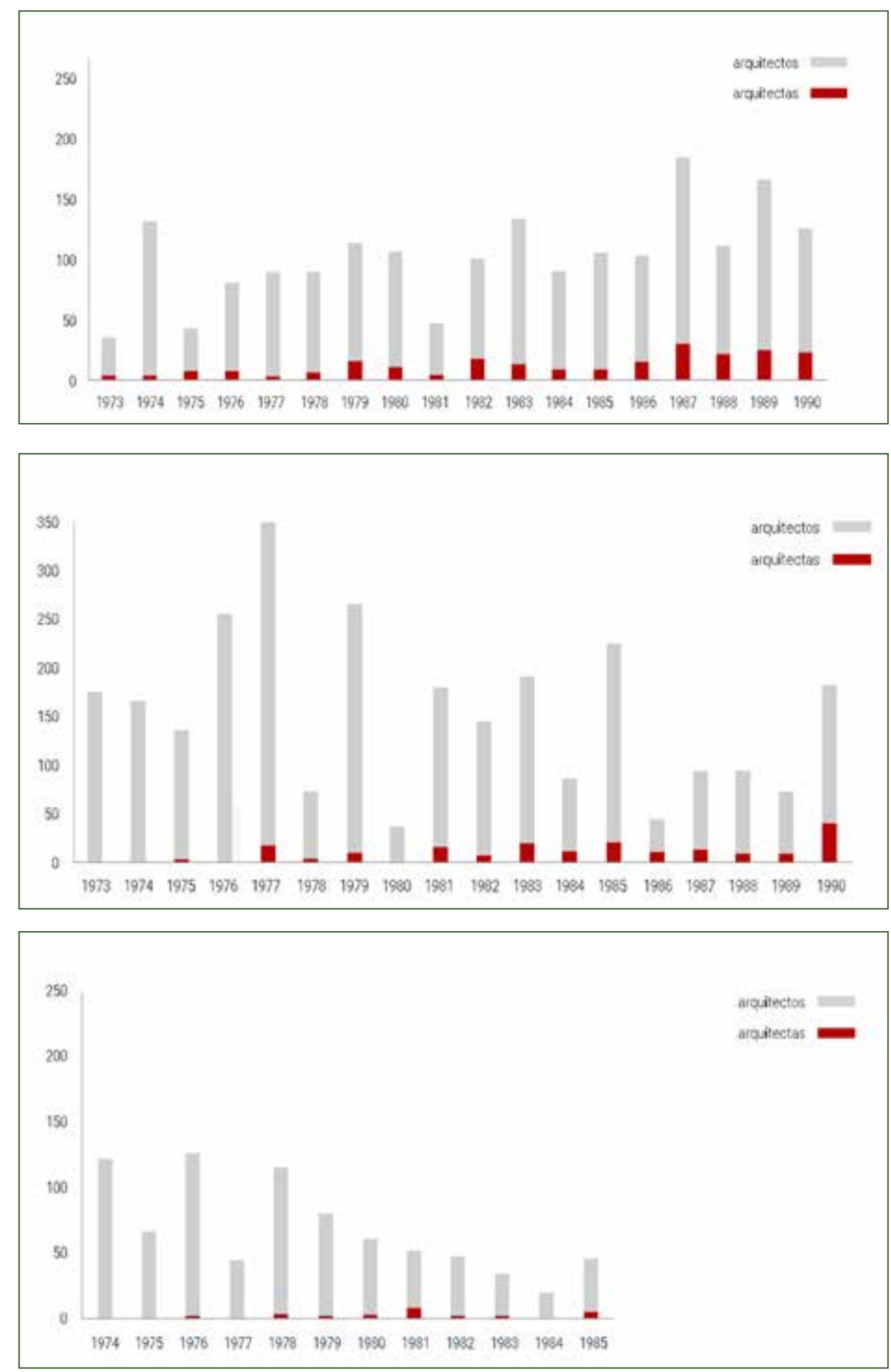

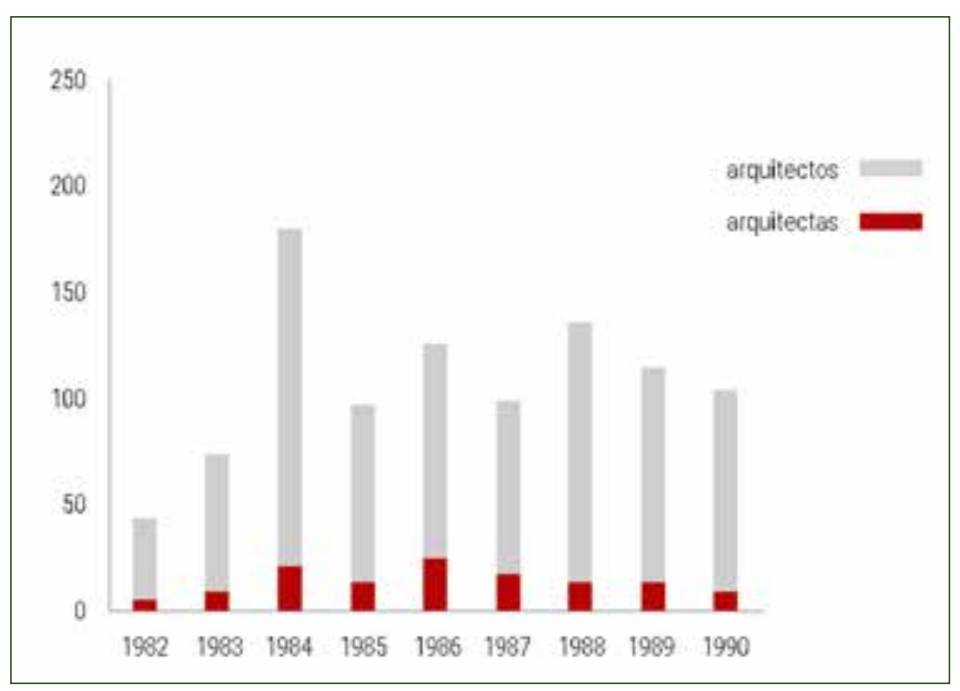

Figura 4: Presencia de arquitectos y arquitectas en la revista El Croquis entre 1982 y 1990. Fuente: Elaboración propia. 

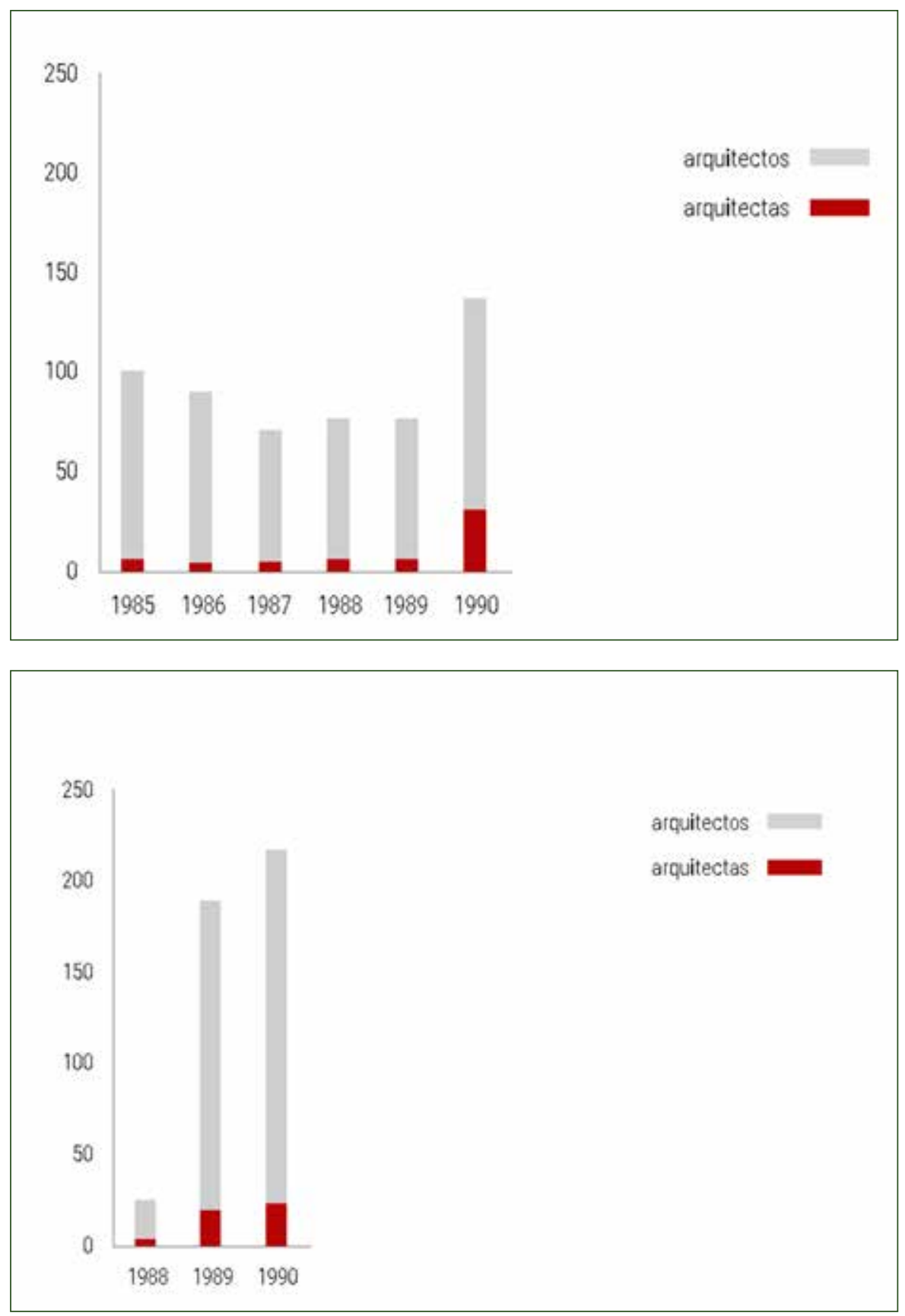

No obstante, hay que tener en cuenta que no todas las profesionales que publicaban en estas revistas eran españolas, siendo necesario desglosar los porcentajes entre profesionales españolas y extrajeras para tener una visión más real de la situación. Así, como muestra la Figura 7, la presencia de profesionales extranjeras en las páginas de estas revistas es considerablemente alta, especialmente a partir de 1979, siendo, incluso, más alta que la de españolas en los últimos años de estudio.

Como resultado del análisis de estos números, aparecen más de un centenar de nombres de arquitectas y urbanistas españolas y extranjeras que de un modo u otro publicaron su trabajo en estas revistas. Aunque a priori este número parece elevado, la mayor parte de estos artículos fueron colaboraciones puntuales, pues tan solo una veintena de arquitectas españolas y tan solo ocho extranjeras vieron su nombre publicado en más de tres ocasiones. Los cuadros inferiores, muestran la relación de profesionales con el mayor número de artículos, por un lado las españolas (Cuadro 2) y por otro las extranjeras (Cuadro 3).
Figura 5: Presencia de arquitectos y arquitectas en la revista AV Monografías entre 1985 y 1990. Fuente: Elaboración propia.

Figura 6: Presencia de arquitectos y arquitectas en la revista Arquitectura Viva entre 1988 y 1990 . Fuente: Elaboración propia. 
Figura 7: Presencia de profesionales españolas y extranjeras en las revistas estudiadas entre 1973 y 1990. Fuente: Elaboración propia.

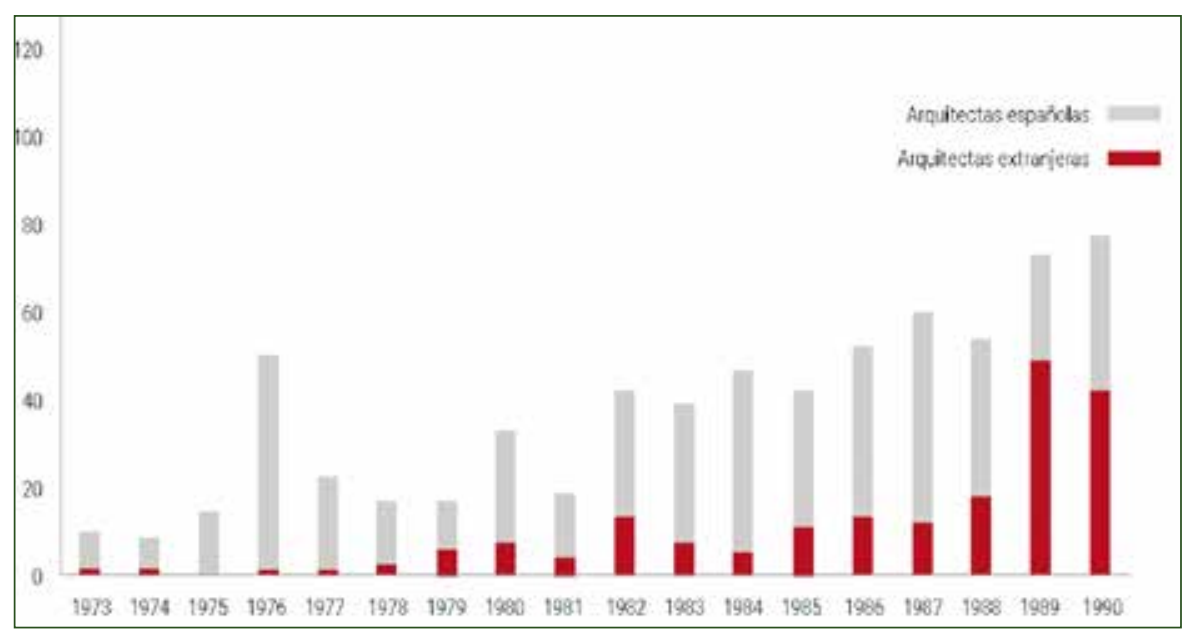

\begin{tabular}{|c|c|c|c|c|c|c|c|}
\hline & $\begin{array}{c}\text { Arquitectura } \\
\text { Viva }\end{array}$ & $\begin{array}{c}A V \\
\text { Monografias }\end{array}$ & El Croquis & Arquitectura & Bis & Cuadernos & TOTAL \\
\hline Cronología estudiada & $(1988-1990)$ & $(1985-1990)$ & $(1982-1990)$ & $(1973-1990)$ & $(1974-1985)$ & $(1973-1990)$ & $(1973-1990)$ \\
\hline $\begin{array}{l}\text { N. }{ }^{\circ} \text { de revistas } \\
\text { estudiadas }\end{array}$ & 15 & 26 & 47 & 118 & 52 & 93 & 351 \\
\hline $\begin{array}{l}\mathrm{N} .^{\circ} \text { total de artículos/ } \\
\text { revista }\end{array}$ & 386 & 495 & 850 & 1.633 & 785 & 2574 & 6723 \\
\hline Carme Pinós & 1 & 1 & 16 & 2 & - & 4 & 24 \\
\hline Roser Amadó & 1 & 2 & 2 & 4 & 2 & 11 & 22 \\
\hline$M^{a}$. Teresa Muñoz & - & - & - & 15 & 6 & - & 21 \\
\hline Marta Cervelló & - & - & - & - & - & 15 & 15 \\
\hline Sara de la Mata & - & - & 1 & 12 & - & - & 13 \\
\hline Pepita Teixidor & - & - & 1 & - & - & 12 & 13 \\
\hline 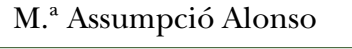 & - & - & - & - & - & 10 & 10 \\
\hline Beth Galí i Camprubí & - & - & 1 & - & 2 & 7 & 10 \\
\hline Mercedes Reig & 8 & 1 & - & - & - & - & 9 \\
\hline Fuensanta Nieto & & - & 1 & 7 & - & - & 8 \\
\hline $\begin{array}{l}\text { María Aroca } \\
\text { Hernández-Ros }\end{array}$ & - & - & 5 & 3 & - & - & 8 \\
\hline Carmen Bravo Durá & - & - & 3 & 5 & - & - & 8 \\
\hline Ana Bohigas & - & - & 2 & 2 & - & 3 & 7 \\
\hline Carme Fiol & - & - & - & 2 & - & 4 & 6 \\
\hline $\begin{array}{l}\text { M. }{ }^{a} \text { José Aranguren } \\
\text { López }\end{array}$ & - & - & 2 & 3 & - & - & 5 \\
\hline $\begin{array}{l}\text { Carmen Herrero } \\
\text { Izquierdo }\end{array}$ & - & - & 1 & 4 & - & - & 5 \\
\hline Marta Pujol & - & - & 3 & 2 & - & - & 5 \\
\hline Mercedes Trias de Bes & - & - & 3 & - & - & 2 & 5 \\
\hline $\mathrm{M}^{\mathrm{a}}$ Pilar de la Villa Ugas & - & 1 & - & - & - & 4 & 5 \\
\hline Rosa Barbá & - & - & - & - & - & 5 & 5 \\
\hline $\begin{array}{l}\text { María Casariego } \\
\text { Córdora }\end{array}$ & - & - & 1 & 3 & - & - & 4 \\
\hline
\end{tabular}


Referentes femeninos en la cultura arquitectónica española. Las revistas especializadas como herramienta de análisis...

\begin{tabular}{|c|c|c|c|c|c|c|c|}
\hline & $\begin{array}{c}\text { Arquitectura } \\
\text { Viva }\end{array}$ & $\begin{array}{c}A V \\
\text { Monografias }\end{array}$ & El Croquis & Arquitectura & Bis & Cuadernos & TOTAL \\
\hline M. ${ }^{a}$ Luisa López Sardá & - & - & 1 & 1 & 2 & - & 4 \\
\hline $\begin{array}{l}\text { Soledad Madrilejos } \\
\text { Fernández }\end{array}$ & - & - & 2 & 2 & - & - & 4 \\
\hline Marta Maíz Apellániz & - & - & 3 & 1 & - & - & 4 \\
\hline Miriam Pascual Lujan & - & - & - & 4 & - & - & 4 \\
\hline
\end{tabular}

Cuadro 2: Relación de arquitectas españolas y su número de apariciones en las diferentes revistas. Fuente: Elaboración propia.

\begin{tabular}{|c|c|c|c|c|c|c|c|}
\hline & $\begin{array}{c}\text { Arquitectura } \\
\text { Viva }\end{array}$ & $\begin{array}{c}A V \\
\text { Monografias }\end{array}$ & El Croquis & Arquitectura & Bis & Cuadernos & TOTAL \\
\hline Cronología estudiada & $(1988-1990)$ & $(1985-1990)$ & $(1982-1990)$ & $(1973-1990)$ & $(1974-1985)$ & $(1973-1990)$ & $(1973-1990)$ \\
\hline $\begin{array}{l}\mathrm{N}^{\circ} \text { total de artículos/ } \\
\text { revista }\end{array}$ & 386 & 495 & 850 & 1633 & 785 & 2574 & 6723 \\
\hline Marta Thorne & 1 & - & - & 3 & - & 12 & 16 \\
\hline Liane Lefaivre & 1 & 4 & 1 & 1 & - & - & 7 \\
\hline Bárbara W. Balluffi & - & - & 2 & 4 & - & - & 6 \\
\hline Marta Schawrtz & - & - & - & 1 & - & 5 & 6 \\
\hline Gae Aulenti & - & 2 & - & 1 & 1 & - & 4 \\
\hline Franziska Bollerey & - & 4 & - & - & - & - & 4 \\
\hline Alison Smithson & - & - & 1 & - & 2 & 1 & 4 \\
\hline Marina Waisman & 3 & 1 & - & - & - & - & 4 \\
\hline Anne Greenwald & - & 3 & - & - & - & - & 3 \\
\hline Mary Margaret Jones & - & - & - & - & - & 3 & 3 \\
\hline Susan Narduli & - & 3 & - & - & - & - & 3 \\
\hline Lisa Roth & - & - & - & - & - & 3 & 3 \\
\hline Bárbara Tietze & - & 3 & - & - & - & - & 3 \\
\hline Sharon Williams & - & 3 & - & - & - & - & 3 \\
\hline Cynthia Weese & - & - & - & 3 & - & - & 3 \\
\hline Phoebe Wall & - & - & - & 2 & - & - & 2 \\
\hline Kristina Hartmann & - & 2 & - & - & - & - & 2 \\
\hline Catherine Cooke & 2 & - & - & - & - & - & 2 \\
\hline Sylvia Lavin & 1 & 1 & - & - & - & - & 2 \\
\hline Doriana O. Mandrelli & 2 & - & - & - & - & - & 2 \\
\hline Silvia Pizzocaro & - & - & 2 & - & - & - & 2 \\
\hline Jaqueline Robertson & - & - & - & 1 & - & 1 & 2 \\
\hline Suzanne Stephens & - & - & 1 & - & 1 & - & 2 \\
\hline Ursula Spitz & - & - & - & - & - & 2 & 2 \\
\hline Laura Thermes & - & - & - & 2 & - & - & 2 \\
\hline
\end{tabular}

Entre las profesionales españolas aparecen tres nombres principales: Carme Pinós, Roser Amadó y María Teresa Muñoz (Cuadro 2); entre las extranjeras destacan Martha Thorne, Liane Lefaivre, Bárbara W.
Cuadro 3: Relación de arquitectas extranjeras y su número de apariciones en las diferentes revistas. Fuente: Elaboración propia. 
Balluffi y Marta Schawrtz (Cuadro 3). El total de las publicaciones dedicadas a Carme Pinós representa un 0,36\% del conjunto de artículos publicados, el de María Teresa Muñoz un 0,34\% y el de Roser Amadó un $0,31 \%$. El resto de nombres femeninos listados representan un porcentaje todavía menor. Como vemos, la visibilidad del trabajo realizado por mujeres es muy bajo comparado con el de colegas masculinos. Asimismo, es poco representativo del creciente porcentaje de profesionales mujeres que habían realizado o estaban realizando estudios de arquitectura en estos mismos años, lo cual abre un nuevo camino para investigaciones futuras.

Como se ha comentado anteriormente, Roser Amadó fue uno de los primeros referentes femeninos ya reconocido en textos históricos escritos en los noventa. Amadó se tituló en la Escuela de Barcelona en 1968 y, en este estudio, sobresale con 22 publicaciones ligadas a su colega Lluís Domènech, con quien compartía estudio de arquitectura. En los años objeto de estudio su trabajo se vio publicado en todas las revistas de arquitectura especializadas estudiadas, aunque alcanzó mayor difusión en la publicación de Quaderns d'arquitectura $i$ urbanisme, con proyectos como el 'Pla especial de reforma interior del Centre de Lleida' (Amadó, 1982).

Por otro lado, destaca Carme Pinós, arquitecta titulada en 1978 por la Escuela de Arquitectura de Barcelona. Pinós desarrollaba su carrera profesional en estos años junto a su socio, Enric Miralles, con el que compartió 24 artículos en torno a su obra arquitectónica. Además, el estudio Miralles-Pinós contó con un número monográfico en la revista El Croquis en 1987, siendo Carme Pinós la primera arquitecta española en tener este reconocimiento. La obra de arquitectura que destacó en las diferentes publicaciones fue el concurso y proyecto para el Cementerio de Igualada, un proyecto que se desarrolló entre 1985 y 1994.

El tercero de los nombres sobresalientes es el de María Teresa Muñoz. Su caso es significativo ya que los artículos publicados son de crítica, algo en plena consonancia con el creciente interés de los arquitectos y arquitectas por la historia y la teoría de arquitectura, como señalaba Antón Capitel. Muñoz se tituló en la Escuela de Arquitectura de Madrid en 1972 y continuó su educación en Canadá, en la Universidad de Toronto. A su vuelta a Madrid destacó por su participación como crítica de arquitectura en las revistas Arquitecturas Bis y Arquitectura. Además, a finales de los años setenta, y junto a un grupo de colegas arquitectos, dirigió la revista madrileña Arquitectura, siendo la primera mujer en participar de manera activa en la toma de decisiones de esta prestigiosa revista. Así, y probablemente derivado del auge editorial de la época, no parece extraño que las siguientes profesionales españolas destacadas aparezcan ligadas al ámbito editorial. Marta Cervelló cuenta con 15 artículos en la revista Cuadernos de Arquitectura y Urbanismo en la que colaboró de 1986 a 1991; Sara de la Mata, colaboradora en Arquitectura, publicó 13 artículos, la mayoría en esta revista; y Mercedes Reig resalta, como crítica en Arquitectura Viva, con 9 publicaciones.

El ámbito de la literatura en arquitectura también sobresale en lo que se refiere a las profesionales extranjeras con repercusión en estas revistas españolas. Por un lado, se distingue la norteamericana Martha Thorne, que en estos años colaboró con la revista Quaderns d'arquitectura i urbanisme, en este caso con una serie de artículos y entrevistas a arquitectos publicadas entre 1982 y 1986. Por otro lado, sobresalen artículos de la arquitecta austríaca Liane Lefaivre en torno a las ideas asociadas al 'Regionalismo crítico', en ocasiones junto a Alexander Tzonis. El 
despunte que parece tener la crítica de arquitectura como ámbito de trabajo de profesionales femeninas propiciaría realizar un estudio más detallado en torno a esta cuestión, es decir, parece necesario analizar el tipo de especialización y el ámbito de trabajo al que se estaban dedicando las diferentes profesionales nombradas. Así, se ha realizado un desglose identificando si el modo en el que se visibiliza su trabajo como autora principal de un proyecto de arquitectura o de urbanismo, como autora de artículos de historia o de crítica de arquitectura, o como colaboradora en un equipo de arquitectura.

\begin{tabular}{|c|c|c|c|c|}
\hline \multirow[b]{2}{*}{ Carme Pinós } & \multirow{2}{*}{$\begin{array}{c}\text { Proyectos } \\
23\end{array}$} & \multirow{2}{*}{$\begin{array}{c}\text { Crítica } \\
1\end{array}$} & \multicolumn{2}{|c|}{ Colaboradora } \\
\hline & & & - & 24 \\
\hline Roser Amadó & 20 & 1 & 1 & 22 \\
\hline M. ${ }^{\mathrm{a}}$ Teresa Muñoz & 1 & 19 & 1 & 21 \\
\hline Marta Cervelló & - & 15 & - & 15 \\
\hline Sara de la Mata & 2 & 11 & - & 13 \\
\hline Pepita Teixidor & 5 & 7 & 1 & 13 \\
\hline M. ${ }^{\mathrm{a}}$ Assumpció Alonso & - & 10 & - & 10 \\
\hline Beth Galí i Camprubí & 9 & 9 & & 10 \\
\hline Mercedes Reig & - & 8 & - & 9 \\
\hline Fuensanta Nieto & - & - & - & 8 \\
\hline María Aroca Hernández-Ros & 8 & - & - & 8 \\
\hline Carmen Bravo Durá & 8 & - & - & 8 \\
\hline Ana Bohigas & - & - & 7 & 7 \\
\hline Carme Fiol & 6 & - & - & 6 \\
\hline M. José Aranguren López & 5 & - & - & 5 \\
\hline Carmen Herrero Izquierdo & 3 & - & 2 & 5 \\
\hline Marta Pujol & - & - & 5 & 5 \\
\hline Mercedes Trias de Bes & 1 & - & 4 & 5 \\
\hline M. ${ }^{\text {a }}$ Pilar de la Villa Ugas & 5 & - & - & 5 \\
\hline Rosa Barba & 1 & 2 & 2 & 5 \\
\hline María Casariego Córdora & 2 & - & 2 & 4 \\
\hline 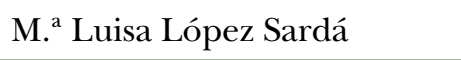 & 4 & - & - & 4 \\
\hline $\begin{array}{l}\text { Soledad Madrilejos } \\
\text { Fernández }\end{array}$ & 4 & - & - & 4 \\
\hline Marta Maíz Apellániz & 4 & - & - & 4 \\
\hline Miriam Pascual Lujan & 4 & - & - & 4 \\
\hline TOTAL & 115 & 83 & 25 & \\
\hline
\end{tabular}

Cuadro 4: Relación de arquitectas españolas y el modo en el que se visibiliza su trabajo en las diferentes revistas. Fuente: Elaboración propia. 
Cuadro 5: Relación de arquitectas extranjeras y el modo en el que se visibiliza su trabajo en las diferentes revistas. Fuente: Elaboración propia.

\begin{tabular}{|c|c|c|c|c|}
\hline & Proyectos & Crítica & \multicolumn{2}{|c|}{ Colaboradora } \\
\hline Marta Thorne & 1 & 15 & - & 16 \\
\hline Liane Lefaivre & - & 7 & - & 7 \\
\hline Bárbara W. Balluffi & 2 & - & 4 & 6 \\
\hline Marta Schawrtz & 6 & - & - & 6 \\
\hline Gae Aulenti & 2 & 2 & - & 4 \\
\hline Franziska Bollerey & - & 4 & - & 4 \\
\hline Alison Smithson & 1 & 3 & - & 4 \\
\hline Marina Waisman & - & 4 & - & 4 \\
\hline Anne Greenwald & - & - & 3 & 3 \\
\hline Mary Margaret Jones & 3 & - & - & 3 \\
\hline Susan Narduli & - & - & 3 & 3 \\
\hline Lisa Roth & 3 & - & - & 3 \\
\hline Bárbara Tietze & - & 3 & - & 3 \\
\hline Sharon Williams & - & - & 3 & 3 \\
\hline Cynthia Weese & 2 & 1 & - & 3 \\
\hline Phoebe Wall & 1 & 1 & - & 2 \\
\hline Kristina Hartmann & - & 2 & - & 2 \\
\hline Catherine Cooke & - & 2 & - & 2 \\
\hline Sylvia Lavin & - & 2 & - & 2 \\
\hline Doriana O. Mandrelli & - & 2 & - & 2 \\
\hline Silvia Pizzocaro & - & 2 & - & 2 \\
\hline Jaqueline Robertson & 1 & 1 & - & 2 \\
\hline Suzanne Stephens & - & 2 & - & 2 \\
\hline Ursula Spitz & 2 & - & - & 2 \\
\hline Laura Thermes & - & 2 & - & 2 \\
\hline TOTAL & 24 & 55 & 13 & \\
\hline
\end{tabular}

Como muestran los Cuadros 4 y 5 , los artículos escritos por mujeres destacan considerablemente, tanto en el caso de profesionales españolas como extranjeras. Esto supone que durante el periodo comprendido entre 1973 al 1990 las diferentes profesionales tienen una mayor presencia en estas revistas especializadas lo hacen como críticas de arquitectura. Además, según los datos, ellas suelen publicar más escritos en solitario que en colaboración con un colega masculino. Atendiendo a su evolución temporal, es significativo que el número de artículos escritos por mujeres en solitario asciende año a año, concretamente de un 1,3\% en 1973 a un 12,7\% en 1990; no obstante, en su mayoría versan sobre la labor desarrollada por arquitectos. Es significativo que los textos de corte teórico que tienen como objeto de análisis la obra arquitectónica de una profesional mujer son prácticamente inexistentes - un primer artículo dedicado a la carrera arquitectónica de una mujer, Eileen Gray, aparece en la revista Arquitecturas Bis (Martín de Terán, 1976). 
Los datos son similares en lo que se refiere a la presencia de proyectos de arquitectura y urbanismo de autoría femenina publicados en estos medios. Mientras que en el análisis de los escritos de crítica se observa un porcentaje de visibilidad de alrededor de un 20\%, los artículos sobre proyectos arquitectónicos y urbanísticos suponen un $24 \%$ del total de obras publicadas. No obstante, es necesario mantener cierta cautela con el análisis de estos datos ya que puede resultar engañoso al ser gran parte de las profesionales femeninas colaboradoras, y no autoras, de los proyectos de arquitectura y urbanismo reseñados. Es el caso de arquitectas como Ana Bohigas, que aparece en 7 ocasiones, únicamente en colaboración con los arquitectos Luís Clotet y Oscar Tusquets; o el de Mercedes Trías de Bes, que lo hace como parte del equipo de Carlos Ferrater. Este modo de trabajo no queda, en muchas ocasiones, documentado de la misma manera que cuando se habla directamente de autoras, por lo que es posible que este porcentaje real sea incluso mayor. Normalmente los colaboradores que participan en un proyecto son difícilmente visibles en la documentación de la obra. De esta manera si descartamos del análisis las colaboraciones, la visibilidad de la obra de mujeres desciende, mostrando que estas alcanzan menor difusión en las publicaciones analizadas cuando se trata de visualizar su obra arquitectónica. Una excepción es el caso de Carme Fiol, que junto a María Pilar de la Villa Ugas, son las únicas arquitectas en desarrollar un proyecto en solitario de entre las primeras cincuenta arquitectas listadas. Por otro lado, es significativo que la mayor parte de las profesionales alcanzasen mayor difusión cuando trabajaban dentro de grupos de arquitectos o en pareja con un socio arquitecto. El destacado trabajo de Carme Pinós junto a Enric Miralles, o el de Roser Amadó y Lluís Domènech es reflejo de esta coyuntura. Igualmente aparecen otros referentes femeninos como Fuensanta Nieto cuyo trabajo se desarrolla junto a su socio Enrique Sobejano, el de Soledad Madrilejos junto a su socio Juan Carlos Sancho Osinaga, o el de María José Aranguren junto a su socio José González Gallegos. Todos ellos muestran una gran proyección dentro del marco temporal estudiado y que, por su conocido impacto actual, se puede predecir que tomará un impulso considerable en la siguiente década.

\section{Conclusión: Hacia una construcción de referentes femeninos en la cultura arquitectónica española}

Como puede observarse, la presencia del trabajo realizado por arquitectas y urbanistas en las revistas de arquitectura entre 1973 y 1990 es escaso, con porcentajes globales inferiores al $0,5 \%$. No obstante, estas mujeres fueron las primeras en romper ese 'techo de cristal' que es la presencia femenina en los medios y en la cultura arquitectónica española. Este estudio, como primer acercamiento a esta temática, pretende arrojar luz a esta cuestión y proponer referentes para investigaciones futuras. Sería necesario ampliar el estudio a la totalidad de revistas de arquitectura de la época hasta la actualidad para tener un análisis global de la presencia de mujeres en los medios especializadas. No obstante, este trabajo ya nos permite identificar varias figuras femeninas relevantes. Algunos de estos nombres destacados los podríamos presuponer antes de hacer este estudio estadístico por la repercusión actual de su trayectoria, como es el caso de la arquitecta catalana Carme Pinós cuya obra ha sido premiada y reconocida internacionalmente. Asimis- 
mo, nombres como el de Roser Amadó también eran esperados antes de realizar el estudio, dada su presencia en textos históricos, como el ya citado de Antón Capitel, aunque este análisis permite objetivar su visibilidad real en el periodo de estudio concreto. No obstante, es resenable que ambas aparecen en los medios ligados a un socio masculino, lo que pone sobre la mesa la falta de visibilidad de trabajos realizados en solitario por mujeres. Esta cuestión no ocurre en el ámbito de la crítica de arquitectura, en el que sí vemos una mayor visibilidad de textos escritos únicamente por una mujer, como es el caso de María Teresa Muñoz o Martha Thorne, entre otras. Ambas cuestiones parecen necesitar una mayor investigación que ayude a entender los mecanismos de toma de decisiones de los consejos editoriales de las diferentes revistas de arquitectura y permita entender el porqué de esa situación. Asimismo, esta metodología propone un alto número de nombres menos conocidos en la actualidad cuya labor sería deseable estudiar con mayor profundidad para un mejor entendimiento de la realidad social de la época. Con todo, la necesidad de desarrollar nuevos estudios académicos y actividades culturales que permitan contextualizar la aportación de estas figuras a la cultura arquitectónica española se torna como una tarea importante para futuras investigaciones. Una tarea que se presenta como una asignatura pendiente y necesaria para conseguir crear modelos femeninos en los que jóvenes arquitectas, arquitectos y urbanistas del presente siglo xx puedan buscar referentes de ejemplaridad y liderazgo y entender ambas disciplinas en términos de igualdad. 


\section{Referencias}

Abril Navarro, M.V. y Maranda López, M.J. (1971, 5 de marzo). Familia y trabajo de la mujer. Triunfo, $653,28-29$.

Agudo Arroyo, Y. y Sánchez de Madariaga, I. (2011). Construyendo un lugar en la profesión: trayectorias de las arquitectas españolas. Feminismo/s, 17, 155-181.

Amadó, R. (1982). Pla especial de reforma interior del Centre de Lleida. Quaderns d'arquitectura i urbanis$m e, 155,44-48$.

Ansón, A. (2010). Televisión y literatura en la España de la Transición (1973-1982). Zaragoza: Institución Fernando el Católico.

Arnell, S. y García Castañeda, S. (1988). La cultura española en el postfranquismo: diez años de cine, cultura y literatura en España (1975-1985). Madrid: Playor.

Butler, J. (1990). Gender Trouble: Feminism and the Subversion of Identity. New York: Routledge.

Capitel, A. (1995). Arquitectura Española, 1939-1992. En Summa Artis. Historia del Arte, vol. XL. Madrid: Espasa Calpe.

Falcón O’Neil, L. (1963). Los derechos civiles de la mujer. Barcelona: Colección Nereo.

Falcón O’Neil, L. (1964). Los derechos laborales de la mujer. Madrid: Montecorvo.

Friedan, B. (1965). La mistica de la feminidad. Barcelona: Sagitario.

Larumbe, M.A. (2002). Una inmensa minoría. Influencia y feminismo en la Transición. Zaragoza: Prensas Universitarias de Zaragoza.

Larumbe, M.A. (2004). Las que dijeron no. Palabra y acción del feminismo en la Transición. Zaragoza: Prensas Universitarias de Zaragoza.

Lorde, A. (1984). Age, Race, Class and Sex: Women Redefining Difference. En Sister Outsiders: Essays and Speeches. Freedom: The Crossing Press, pp.114-123.

Martín de Terán, L. (1976). La visita de la vieja dama: Eileen Gray. Arquitectura Bis,16, 7-13.

Moreno, A. (1977). Educación de la mujer: La gran estafa. Vindicación Feminista, 10, 29-37.
Munro, E. (2013). Feminism: A Fourth Wave? Political Insight, 4(2), 22-25. DOI: https://doi. org/10.1111\%2F2041-9066.12021

Muxí, Z., (2013). Primera generación de arquitectas catalanas ETSAB 1964-1975. En Jornadas Mujer y Arquitectura: Experiencia docente, investigadora y profesional. Coruña: Universidade da Coruña, pp. 31-63. Recuperado el 06 de Agosto de 2018, de: https:/ / ruc.udc.es/dspace/bitstream/handle/2183/9986/ JMA_26_27_nov12_ruc.pdf;jsessionid=67F8DD9A2 D6BFA5C702F0A007141B0E4? sequence $=6$.

Rubin, G. (1975). The traffic in Women: Notes on the 'Political Economy' of Sex. En Reiter, R.R. (ed.), Toward an Anthropology of Women. New York: Monthly Review Press, 157-210.

Palacio, M. (2011). El cine y la Transición política en España (1972-1982). Madrid: Biblioteca Nueva.

Pérez-Moreno, L.C. (2016). The 'Transition' as a Turning Point for Female Agency in Spanish Architecture. En Brown, J.B., Harriss, H., Morrow, R. y Soane, J. (eds.), A Gendered Profession: The Question of Representation in Space Making. London: RIBA Publishing, 108-115.

Sánchez de Madariaga, I. (2010). Women in Architecture: the Spanish case. Urban Research E Practice, 3(2), 203-218.

Sánchez de Madariaga, I. (2012). Matilde Ucelay. Una vida en construcción. Madrid: Ministerio de Fomento.

Vilchez, J. (2012). Matilde Ucelay. Primera mujer arquitecta en España [Tesis doctoral]. Universidad de Granada, Granada, España.

Valerie Caven, E. y Navarro-Astor, M. D. (2012). A cross-national study of accommodating and 'usurpatory' practices by women architects in the UK, Spain and France. Architectural Theory Review, 17(23), 365-377.

Wittig, M. (1973). Le Corps Lesbien. Paris: Les Éditions de Minuit.

Pérez-Moreno, L. C. y Santamera, P. (2018). Referentes femeninos en la cultura arquitectónica española. Las revistas especializadas como herramienta de análisis para la valoración de la visibilidad del trabajo realizados por mujeres (1973-1990). Hábitat y Sociedad, 11, 31-47.

<http://dx.doi.org/10.12795/HabitatySociedad.2018.i11.03>

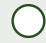




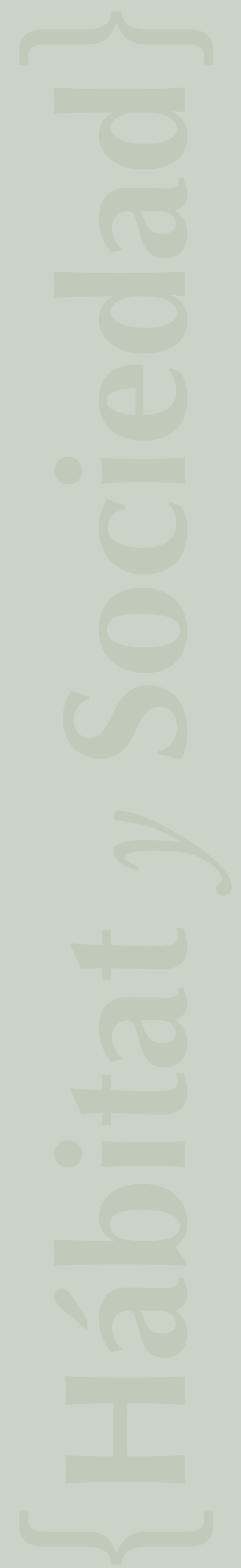

